\title{
A modular absolute bound condition for primitive association schemes
}

\author{
Akihide Hanaki • Ilia Ponomarenko
}

Received: 29 September 2007 / Accepted: 12 June 2008 / Published online: 5 July 2008

(C) Springer Science+Business Media, LLC 2008

\begin{abstract}
The well-known absolute bound condition for a primitive symmetric association scheme $(X, S)$ gives an upper bound for $|X|$ in terms of $|S|$ and the minimal non-principal multiplicity of the scheme. In this paper we prove another upper bounds for $|X|$ for an arbitrary primitive scheme $(X, S)$. They do not depend on $|S|$ but depend on some invariants of its adjacency algebra $K S$ where $K$ is an algebraic number field or a finite field.
\end{abstract}

Keywords Association scheme - Adjacency algebra

\section{Introduction}

Let $(X, S)$ be an association scheme (for a background on association scheme theory we refer to $[1,10]$ and Appendix). Denote by $F S$ its adjacency algebra over a field $F$. As usual we consider $F S$ as a subalgebra of the full matrix algebra $\operatorname{Mat}_{X}(F)$. Set

$$
\operatorname{rk}_{\min }(F, S)=\min _{A \in F S \backslash F J} \operatorname{rk}(A)
$$

where $J$ is the all-one matrix in $F S$ and $\operatorname{rk}(A)$ is the rank of a matrix $A$. One can see that in the commutative case the number $\operatorname{rk}_{\min }(\mathbb{C}, S)$ coincides with the minimal multiplicity $m_{\min }$ of a non-principal irreducible representation of the algebra $\mathbb{C} S$ (see (1)).

Partially supported by RFBR grants 07-01-00485, 08-01-00379 and 08-01-00640. The paper was done during the stay of the author at the Faculty of Science of Shinshu University.

\footnotetext{
A. Hanaki

Faculty of Science, Shinshu University, Matsumoto 390-8621, Japan

e-mail: hanaki@math.shinshu-u.ac.jp

I. Ponomarenko $(\bowtie)$

Petersburg Department of V.A.Steklov Institute of Mathematics, St. Petersburg 191023, Russia

e-mail: inp@pdmi.ras.ru
} 
It is a well-known fact (see [1, Theorem 4.9]) that given a primitive symmetric scheme $(X, S)$ the number $|X|$ can not be arbitrarily large when $|S|$ and $m_{\min }$ are bounded. It was asked there about a reasonable absolute bound condition for an arbitrary primitive commutative scheme. The main goal of this paper is to use the modular representation theory for schemes to get another upper bound for $|X|$ without the assumption of commutativity. Our first result gives the following modular absolute bound condition for primitive schemes.

Theorem 1.1 Let $(X, S)$ be a primitive scheme and let $q$ be a prime power. Set $r=$ $\operatorname{rk}_{\min }\left(\mathbb{F}_{q}, S\right)$. Then

$$
|X| \leq \frac{q^{r}-1}{q-1}
$$

whenever $r>1$. If $r=1$, then $|X|<q$ and $(X, S)$ is a thin scheme of prime order.

We have examples for which the equality holds in Theorem 1.1.

Example 1.2 Let $(X, S)$ be the cyclotomic scheme over a prime field $\mathbb{F}_{p}$ corresponding to its multiplicative subgroup of order $r$. Suppose that there exists a prime $q$ such that $p=\left(q^{r}-1\right) /(q-1)$. Then $\operatorname{rk}_{\min }\left(\mathbb{F}_{q}, S\right)=r$ and the equality in Theorem 1.1 holds. We omit the proof of this fact, but one can easily check it for $(p, r, q)=(31,5,2)$ or $(31,3,5)$.

Given a scheme $(X, S)$ denote by $\mathcal{P}=\mathcal{P}(\mathbb{C} S)$ the set of all central primitive idempotents of the algebra $\mathbb{C} S$. For $P \in \mathcal{P}$ set $m_{P}$ to be the multiplicity of the irreducible representation of $\mathbb{C} S$ corresponding to $P$ in the standard representation (given by the standard $\mathbb{C} S$-module $\mathbb{C} X)$. Put

$$
m_{\min }=\min _{P \in \mathcal{P} \backslash\left\{P_{0}\right\}} m_{P}
$$

where $P_{0}=(1 /|X|) J$ is the principal idempotent of $\mathbb{C} S$. If $(X, S)$ is primitive and $m_{\min }=1$, then it is a thin scheme of prime order (Theorem 2.2). So we may omit this case. Set

$$
\mathbb{Q}(X, S)=\mathbb{Q}\left(\left\{P_{x, y}: P \in \mathcal{P}, x, y \in X\right\}\right) .
$$

It is not necessarily a splitting field of $\mathbb{Q} S$, but it is a Galois extension and every $P \in \mathcal{P}$ belongs to the adjacency algebra $\mathbb{Q}(X, S) S$.

Theorem 1.3 Let $(X, S)$ be a primitive scheme. Suppose that $p$ is a prime which does not divide the Frame number of this scheme (see (A4)) and $m_{\min }>1$. Then

$$
|X| \leq \frac{q^{m_{\min }}-1}{q-1} .
$$

where $q=p^{|\mathbb{Q}(X, S): \mathbb{Q}|}$. 
Remark 1.4 The proof of Theorem 1.3 given in Section 3 shows that the upper bound in (2) can be reduced. For an appropriate $P \in \mathcal{P}$, the bound is

$$
|X| \leq \frac{q^{m_{P}}-1}{q-1}
$$

where $q=p^{\left|\mathbb{Q}\left(\left\{P_{x, y}: x, y \in X\right\}\right): \mathbb{Q}\right|}$.

We do not know any primitive scheme for which the upper bound in (2) is tight. However, there are examples where this bound is less than one given by the absolute bound condition (e.g. for some amorphic primitive schemes $(X, S)$ such that $|X|=q^{2}$ and $|S|=(q+1) / 2$ where $q$ is a prime and $q \neq \equiv 1(\bmod 3)$; in this case $\mathbb{Q}(X, S)=\mathbb{Q}$ and one can take $p=3$ ). On the other hand, one can use inequality (2) to prove the finiteness of some classes of rational primitive schemes (here a scheme is called rational if $\mathbb{Q}$ is a splitting field of its adjacency algebra). In this case, we can apply Theorem 1.3 to any primitive $p^{\prime}$-scheme, that is, a scheme such that the prime $p$ does divide neither $|X|$ nor the valency of an element from $S$.

Corollary 1.5 Given a prime $p$ and a positive integer $r$ the set of rational primitive $p^{\prime}$-schemes for which $m_{\min } \leq r$, is finite.

The class of $p^{\prime}$-schemes with $p=2$ consists of $o d d$ schemes, i.e. those for which only symmetric basis relation of it is a reflexive one. Theorem 1.3 shows that for a fixed $r$ a splitting field of an odd primitive scheme with $m_{\min } \leq r$ grows when $|X|$ grows.

The assumptions of Theorem 1.3 mean that the adjacency algebra of $(X, S)$ over a field of characteristic $p$ is semisimple. Non-semisimple case seems to be much more difficult (see $[6,8])$.

The proofs of Theorems 1.1 and 1.3 are given in Sections 2 and 3 respectively. To make the paper self-contained we put in Appendix the notation and definitions concerning schemes and their adjacency algebras.

Notation As usual by $\mathbb{Q}, \mathbb{C}$ and $\mathbb{F}_{q}$ we denote the fields of rational and complex numbers and a finite field with $q$ elements respectively. Throughout the paper $X$ denotes a finite set. The diagonal of the set $X \times X$ is denoted by $\Delta$. The algebra of all matrices whose entries belong to a field $F$ and whose rows and columns are indexed by the elements of $X$ is denoted by $\operatorname{Mat}_{X}(F)$, the identity matrix by $I$ and the all-one matrix by $J$. Given $A \in \operatorname{Mat}_{X}(F)$ and $x, y \in X$, we denote by $A_{x, y}$ the $(x, y)$ entry of $A$. The Hadamard (componentwise) product of matrices $A, B \in \operatorname{Mat}_{X}(F)$ is denoted by $A \circ B$. The adjacency matrix of a binary relation $r \subset X \times X$ is denoted by $A_{r}$ (this is a $\{0,1\}$-matrix of $\operatorname{Mat}_{X}(F)$ such that $\left(A_{r}\right)_{x, y}=1$ if and only if $\left.(x, y) \in r\right)$. The left standard module of the algebra $\operatorname{Mat}_{X}(F)$ is denoted by $F X$. We will identify the elements of $X$ with the standard basis vectors of $F X$. 


\section{Combinatorics in the adjacency algebra}

First we prove that with any matrix of the adjacency algebra of a scheme one can associate some special relations which are unions of basis relations (a special case of our result also follows from [4, Lemma 4.1]). Namely, let $F$ be a field. Given a matrix $A \in \operatorname{Mat}_{X}(F)$ and an element $\lambda \in F$ we define a binary relation

$$
e_{\lambda}(A)=\{(x, y) \in X \times X: \lambda A x=A y\}
$$

on the set $X$. Clearly, $e_{1}(A)$ is a nonempty equivalence relation on $X$ and $e_{\lambda}(A) \cap$ $e_{\mu}(A)=\emptyset$ for all nonzero elements $\lambda \neq \mu$. Besides, $e_{0}(A)=\emptyset$ if and only if the matrix $A$ has no zero columns. In the latter case, the relation

$$
e(A)=\bigcup_{\lambda \in F} e_{\lambda}(A)
$$

is also an equivalence relation on $X$. Note that $A x$ being the $x$ th column of the matrix $A$ can be considered as an element of $F X$. So $(x, y) \in e(A)$ if and only if the vectors $A x, A y \in F X$ are linearly dependent.

In [4, Lemma 4.1] it was proved that given a scheme $(X, S)$ and a matrix $A \in \mathbb{C} S$ the relation $e_{\lambda}(A)$ with $\lambda=1$ belongs to the set $S^{*}$ of all unions of relations from $S$. The following statement generalizes this result for an arbitrary field and all $\lambda$ 's. Below we denote by $A_{e}$ and $A_{\lambda}$ the adjacency matrices of the relations $e(A)$ and $e_{\lambda}(A)$ respectively. In the first part of the proof we follow [3, Lemma 1.42].

Theorem 2.1 Let $(X, S)$ be a scheme and let $F$ be a field. Then $e_{\lambda}(A) \in S^{*}$ for all $A \in F S$ and $\lambda \in F$.

Proof Without loss of generality we assume that $A \neq 0$. First we suppose that $F=\mathbb{C}$. Since $A \in \mathbb{C} S$, we also have $A^{*} \in \mathbb{C} S$ where $A^{*}$ is the Hermitian conjugate of $A$. This implies that $A^{*} A \in \mathbb{C} S$. So given $x \in X$ the number $\left(A^{*} A\right)_{x, x}$ equals to the coefficient of the identity matrix $I=A_{\Delta}$ in the decomposition of the matrix $A^{*} A$ by the matrices $A_{s}, s \in S$. Denote it by $d$. Then by the Cauchy-Schwarz inequality we conclude that

$$
\left|\left(A^{*} A\right)_{x, y}\right|=|\langle A x, A y\rangle| \leq\|A x\| \cdot\|A y\|=d
$$

where $\langle\cdot, \cdot\rangle$ and $\|\cdot\|$ are the inner product and the Euclidean norm in $\mathbb{C} X$ respectively. Moreover, the equality in (4) is attained if and only if the vectors $A x$ and $A y$ are linearly dependent. Thus $\left|\left(A^{*} A\right)_{x, y}\right|=d$ if and only if $(x, y) \in e(A)$. Due to (A2) this shows that $A_{e} \in \mathbb{C} S$ and so $e(A) \in S^{*}$. On the other hand, given $(x, y) \in e_{\lambda}(A)$ the number

$$
\left(A^{*} A\right)_{x, y}=\langle A x, A y\rangle=\langle A x, \lambda A x\rangle=\lambda\langle A x, A x\rangle=\lambda d .
$$

does not depend on $(x, y)$. By (3) this means that

$$
\left(A^{*} A\right) \circ A_{e}=d \sum_{\lambda \in \Lambda} \lambda A_{\lambda}
$$


where $\Lambda=\left\{\lambda \in \mathbb{C}: e_{\lambda}(A) \neq \emptyset\right\}$. Since the matrices $A^{*} A$ and $A_{e}$ belong to $\mathbb{C} S$, we conclude by (A2) that $A_{\lambda} \in \mathbb{C} S$ and hence $e_{\lambda}(A) \in S^{*}$.

Let $F$ be an arbitrary field. Since $A \in F S$, any two columns of $A$ consist of the same elements of $F$. Denote the set of all of them by $M$. Then

$$
M \lambda=M, \quad \lambda \in \Gamma,
$$

where $\Gamma=\left\{\lambda \in F: e_{\lambda}(A) \neq \emptyset\right\}$. Easily we can see that $\Gamma$ is a finite subgroup of the multiplicative group $F^{\times}$, and so $\Gamma$ is cyclic. Take an injection and a group monomorphism

$$
f: M \rightarrow \mathbb{C}, \mu \mapsto \mu^{\prime}, \quad \varphi: \Gamma \rightarrow \mathbb{C}^{\times}, \lambda \mapsto \lambda^{\prime}
$$

such that the permutation groups induced by the actions of $\Gamma$ on $M$, and of $\Gamma^{\prime}=$ $\operatorname{Im}(\varphi)$ on $M^{\prime}=\operatorname{Im}(f)$ are equivalent. Then it is easy to see that

$$
\lambda A x=A y \Leftrightarrow \lambda^{\prime} A^{\prime} x=A^{\prime} y, \quad x, y \in X,
$$

where $A^{\prime} \in \operatorname{Mat}_{X}(\mathbb{C})$ is the complex matrix with entries $A_{x, y}^{\prime}=\left(A_{x, y}\right)^{f}$ for all $x, y$. So $e(A)=e\left(A^{\prime}\right)$ and we are done by the first part of the proof.

It was proved in $[9$, p. 71] that any primitive scheme having a nonreflexive basis relation of valency 1 is a thin scheme of prime order. The following theorem gives a "dual" version of this result.

Theorem 2.2 Let $(X, S)$ be a primitive scheme and let $F$ be a field. Suppose that $\operatorname{rk}_{\min }(F, S)=1$. Then $(X, S)$ is a thin scheme of prime order.

Proof $\operatorname{Since} \mathrm{rk}_{\min }(\mathbb{F}, S)=1$, there exists a rank 1 matrix $A \in F S \backslash F J$. This implies that any two columns of $A$ are linear dependent. So $e(A)=X \times X$. On the other hand, $e_{1}(A) \in S^{*}$ by Theorem 2.1. Due to the primitivity of $(X, S)$ this implies that $e_{1}(A) \in\{\Delta, X \times X\}$. Moreover, since $A \notin F J$, we see that $e_{1}(A)=\Delta$. Thus by formula (3) we conclude that

$$
A=\sum_{x \in X} \lambda_{x} A_{\lambda_{x}}
$$

for some $\lambda_{x} \in F$ such that $\lambda_{x} \neq \lambda_{y}$ for all $x \neq y$. So $e_{\lambda_{x}}(A) \in S$ and the valency of $e_{\lambda_{x}}(A)$ equals 1 for all $x \in X$ (see (A2)). This shows that the scheme $(X, S)$ is thin. To complete the proof it suffices to note that any primitive thin scheme is of prime order.

Now we can prove Theorem 1.1.

Proof of Theorem 1.1 From the hypothesis it follows that there exists a rank $r$ matrix $A \in \mathbb{F}_{q} S \backslash \mathbb{F}_{q} J$. By Theorem 2.1 we know that $e(A), e_{1}(A) \in S^{*}$. Since the scheme $(X, S)$ is primitive, this implies that

$$
e(A), e_{1}(A) \in\{\Delta, X \times X\} .
$$


However, since $A$ is not a multiple of $J$, it follows that $e_{1}(A)=\Delta$, and hence

$$
|X|=|\{A x: x \in X\}| .
$$

On the other hand, if $e(A)=X \times X$, then any two vectors $A x$ and $A y$ are linearly dependent. So $r=\operatorname{rk}(A)=1$ and $|\{A x: x \in X\}| \leq\left|F_{q}^{\times}\right|=q-1$. By (6) this proves the second part of the theorem. Thus without loss of generality we can assume that $e(A)=\Delta$. Then any two distinct vectors $A x$ and $A y$ are linearly independent. This means that $r=\operatorname{rk}(A)>1$ and

$$
|\{A x: x \in X\}| \leq \frac{q^{r}-1}{q-1},
$$

and we are done by (6).

\section{Matrix rank in the adjacency algebra}

In this section we deduce Theorem 1.3 from Theorem 1.1. To do this, we will consider adjacency algebras over an algebraic number field and its ring of integers. We refer to [7] for standard facts from algebraic number theory. For the rest of the section we fix a scheme $(X, S)$, an algebraic number field $K$ and a rational prime number $p$.

Denote by $R$ the ring of integers of $K$. Take its prime ideal $\mathfrak{P}$ lying above $p \mathbb{Z}$ and set $f$ to be the degree of $\mathfrak{P}$. Then

$$
f \leq|K: \mathbb{Q}|
$$

and the quotient ring $R / \mathfrak{P}$ is isomorphic to the field $\mathbb{F}_{q}$ where $q=p^{f}$. Denote by $K_{\mathfrak{P}}$ and $R_{\mathfrak{P}}$ the $\mathfrak{P}$-adic field and the ring of $\mathfrak{P}$-adic integers respectively. Then

$$
R_{\mathfrak{P}}=\left\{a \in K_{\mathfrak{P}}: \nu_{\mathfrak{P}}(a) \geq 0\right\}
$$

where $\nu_{\mathfrak{P}}$ is the $\mathfrak{P}$-valuation on $K_{\mathfrak{P}}$. Here $\nu_{\mathfrak{P}}(a)=\infty$ if and only if $a=0$. Since $R_{\mathfrak{P}} / \mathfrak{P} R_{\mathfrak{P}} \cong R / \mathfrak{P}$, the ring epimorphism $R \rightarrow \mathbb{F}_{q}$ induces the epimorphism $R_{\mathfrak{P}} \rightarrow$ $\mathbb{F}_{q}, a \mapsto \bar{a}$, and hence the epimorphism

$$
R_{\mathfrak{P}} S \rightarrow \mathbb{F}_{q} S, \quad \sum_{s \in S} a_{s} A_{s} \mapsto \sum_{s \in S} \overline{a_{s}} A_{s}
$$

where we use the natural identification of $\{0,1\}$-matrices in $R_{\mathfrak{P}} S$ and $\mathbb{F}_{q} S$. The image of $A \in R_{\mathfrak{P}} S$ is denoted by $\bar{A}$.

Lemma 3.1 Suppose $\mathbb{F}_{q} S$ is semisimple. Then every central idempotent of $K_{\mathfrak{P}} S$ belongs to $R_{\mathfrak{P}} S$. 
Proof Let $P$ be a central idempotent of $K_{\mathfrak{P}} S$. Without loss of generality we assume that $P \neq 0$. Then due to (8) it suffices to verify that $v(P)=0$ where $v=v_{\mathfrak{P}}$ and given an element $A=\sum_{s \in S} a_{s} A_{s}$ of the algebra $K_{\mathfrak{P}} S$ we set

$$
v(A)=\min _{s \in S} v\left(a_{s}\right) .
$$

Clearly, $v(A B) \geq v(A)+v(B)$ and $v(a A)=v(a)+v(A)$ for all $A, B \in K_{\mathfrak{P}} S$ and $a \in K_{\mathfrak{P}}$. So

$$
v(P)=v\left(P^{2}\right) \geq v(P)+v(P)
$$

whence it follows that $v(P) \leq 0$ (here $v(P)<\infty$ because $P \neq 0$ ). Suppose that $v(P)<0$. Set $Q=a P$ where $a$ is an element of $K_{\mathfrak{P}}$ such that $v(Q)=v(a)+v(P)=$ 0 . Then $\bar{Q} \neq 0$ (see (9)) and

$$
v\left(Q^{2}\right)=v\left(a^{2} P\right)=v(a)+(v(a)+v(P))=v(a)=-v(Q)>0 .
$$

So $\overline{Q^{2}}=0$. Since $\bar{Q}$ is in the center of the algebra $\mathbb{F}_{q} S$, the set $\bar{Q}\left(\mathbb{F}_{q} S\right)$ is a nonzero proper nilpotent ideal of it. However, this contradicts the assumption that $\mathbb{F}_{q} S$ is semisimple.

Remark 3.2 In the proof of Lemma 3.1 we extended the valuation $v_{\mathfrak{P}}$ to the adjacency algebra $K_{\mathfrak{P}} S$ of a scheme $(X, S)$ (see (10)). This extension $v$ has properties: $v(A)=\infty$ iff $A=0, v(A+B) \geq \min (v(A), v(B))$ and $v(A B) \geq v(A)+v(B)$.

Let $P \in \mathcal{P}(\mathbb{C} S)$ be a central primitive idempotent of $\mathbb{C} S$. Then every entry of $P$ is an algebraic number. If the field $K$ contains all entries of $P$, then $P \in K S$ and $K$ can be embedded into both $\mathbb{C}$ and $K_{\mathfrak{P}}$. Through these embeddings, we can regard $P$ as an element of $K_{\mathfrak{P}} S$.

Lemma 3.3 Suppose $\mathbb{F}_{q} S$ is semisimple and the field $K$ contains all entries of a matrix $P \in \mathcal{P}(\mathbb{C} S)$. Then the following statements hold:

(1) $P \in R_{\mathfrak{P}} S$; in particular, the element $\bar{P}$ is defined and belongs to $\mathcal{P}\left(\mathbb{F}_{q} S\right)$,

(2) $\bar{P}\left(\mathbb{F}_{q} S\right) \cong \operatorname{Mat}_{n}\left(\mathbb{F}_{q}\right)$ for some $n$, the irreducible representation of $\mathbb{F}_{q} S$ defined by $\bar{P}$ is absolutely irreducible, and the degree and the multiplicity of it in the standard representation of $\mathbb{F}_{q} S$ coincide with $n_{P}$ and $m_{P}$ respectively (see $\left.(A 3)\right)$.

Proof The first part of statement (1) immediately follows from Lemma 3.1. By [2, Proposition 1.12], we can see that $\bar{P}$ is primitive. Statement (1) is completely proved. Next, since $P$ is primitive in $\mathbb{C} S, \bar{P}$ is primitive in the adjacency algebra over any extension field $E$ of $\mathbb{F}_{q}$, and then $\bar{P}(E S)$ is a simple algebra. Since any finite division ring is a field, the Wedderburn theorem shows that $\bar{P}\left(\mathbb{F}_{q} S\right) \cong \operatorname{Mat}_{n}(F)$ for some $n$ and some finite extension $F$ of $\mathbb{F}_{q}$, and $\bar{P}(F S)$ is also a simple algebra. By the separability of $F$ over $\mathbb{F}_{q}$, we have

$$
\bar{P}(F S) \cong F \otimes_{\mathbb{F}_{q}} \bar{P}\left(\mathbb{F}_{q} S\right) \cong F \otimes_{\mathbb{F}_{q}} \operatorname{Mat}_{n}(F) \cong \operatorname{Mat}_{n}\left(F \otimes_{\mathbb{F}_{q}} F\right)
$$




$$
\cong \operatorname{Mat}_{n}\left(\left|F: \mathbb{F}_{q}\right| F\right) \cong\left|F: \mathbb{F}_{q}\right| \operatorname{Mat}_{n}(F)
$$

Due to the simplicity of $\bar{P}(F S)$ we have $\left|F: \mathbb{F}_{q}\right|=1$ and hence $F=\mathbb{F}_{q}$. This means that the irreducible representation defined by $\bar{P}$ is absolutely irreducible.

Besides, the ranks of the modules

$$
K_{\mathfrak{P}} S=P\left(K_{\mathfrak{P}} S\right) \oplus(I-P)\left(K_{\mathfrak{P}} S\right), \quad \mathbb{F}_{q} S=\bar{P}\left(\mathbb{F}_{q} S\right) \oplus(\bar{I}-\bar{P})\left(\mathbb{F}_{q} S\right)
$$

are the same. Since obviously the ranks of $P\left(K_{\mathfrak{P}} S\right)$ and $(I-P)\left(K_{\mathfrak{P}} S\right)$ do not exceed the ranks of $\bar{P}\left(\mathbb{F}_{q} S\right)$ and $(\bar{I}-\bar{P})\left(\mathbb{F}_{q} S\right)$, respectively, it follows that they are equal. Thus the degrees of irreducible representations corresponding to $P$ and $\bar{P}$ are the same. Also comparing the dimensions of the decompositions of standard modules $K_{\mathfrak{P}} X$ and $\mathbb{F}_{q} X$, we see that the multiplicities of irreducible representations in the standard representations corresponding to $P$ and $\bar{P}$ are the same.

Lemma 3.4 Suppose $\mathbb{F}_{q} S$ is semisimple and the field $K$ contains all entries of $P \in$ $\mathcal{P}(\mathbb{C} S)$. Then there exists $E \in \mathbb{F}_{q} S$ such that $\operatorname{rk}(E)=m_{P}$.

Proof From Lemma 3.3(2), we have $\bar{P}\left(\mathbb{F}_{q} S\right) \cong \operatorname{Mat}_{n_{P}}\left(\mathbb{F}_{q}\right)$. Choose an element $E \in$ $\bar{P}\left(\mathbb{F}_{q} S\right)$ corresponding to a diagonal matrix unit in $\operatorname{Mat}_{n_{P}}\left(\mathbb{F}_{q}\right)$. Since the irreducible representation corresponding to $\bar{P}$ appears $m_{P}$ times in the standard representation, we have that $\operatorname{rk}(E)=m_{P}$.

Now we give a proof of Theorem 1.3.

Proof of Theorem 1.3 Suppose $p$ is not a divisor of the Frame number $\operatorname{Fr}(X, S)$. Then the adjacency algebra of $(X, S)$ over a field of characteristic $p$ is semisimple (see Appendix). Since the field $K=\mathbb{Q}(X, S)$ satisfies the condition of Lemma 3.4 for all $P \in \mathcal{P}(\mathbb{C} S)$, one can find a matrix $E \in \mathbb{F}_{q} S$ such that $\operatorname{rk}(E)=m_{\text {min }}$. Since $m_{\text {min }}>1$, we see that $E \notin \mathbb{F}_{q} J$. By Theorem 1.1 and inequality (7) we have

$$
|X| \leq \frac{q^{m_{P}}-1}{q-1}=\frac{p^{m_{P} f}-1}{p^{f}-1} \leq \frac{p^{|K: \mathbb{Q}| m_{P}}-1}{p^{|K: \mathbb{Q}|}-1}
$$

and we are done.

\section{Appendix: Association schemes}

Let $X$ be a finite set and $S$ a partition of $X \times X$ closed with respect to the transpose. A pair $(X, S)$ is called an association scheme or scheme if the reflexive relation $\Delta$ belongs to the set $S$ and given $r, s, t \in S$, the number

$$
c_{r, s}^{t}=|\{z \in X:(x, z) \in r,(z, y) \in s\}|
$$

does not depend on the choice of $(x, y) \in t$. The elements of $S$ and the number $|X|$ are called the basis relations and the order of the scheme. The set of unions of all 
subsets of $S$ is denoted by $S^{*}$. The number $d_{r}=c_{r, r^{*}}^{\Delta}$ where $r^{*}$ is the transpose of $r$, is called the valency of $r$. The scheme $(X, S)$ of order $\geq 2$ is called primitive if any equivalence relation on $X$ belonging to $S^{*}$ coincides with either $\Delta$ or $X \times X$.

Given a field $F$ the linear span $F S$ of the set $\left\{A_{s}: s \in S\right\}$ forms a subalgebra of the algebra $\operatorname{Mat}_{X}(F)$ (see (A1)). This subalgebra is called the adjacency algebra of the scheme $(X, S)$ over $F$. From the definition it follows that $F S$ is closed with respect to the transpose and the Hadamard multiplication. In particular,

$$
a \in F, A \in F S \Rightarrow A^{(a)} \in F S
$$

where $A^{(a)}$ is a $\{0,1\}$-matrix in $\operatorname{Mat}_{X}(F)$ such that $A_{x, y}^{(a)}=1$ if and only if $A_{x, y}=a$. One can see that any $\{0,1\}$-matrix belonging to $F S$ is of the form $A_{s}$ for some $s \in S^{*}$. The set of all central primitive idempotents of the algebra $F S$ is denoted by $\mathcal{P}(F S)$.

The adjacency algebra $\mathbb{C} S$ of the scheme $(X, S)$ over the complex number field $\mathbb{C}$ is semisimple. So by the Wedderburn theorem its standard module $\mathbb{C} X$ is completely reducible. For an irreducible submodule $L$ of $\mathbb{C} X$ corresponding to a central primitive idempotent $P$ of the algebra $\mathbb{C} S$, we set

$$
n_{P}=\operatorname{dim}_{\mathbb{C}}(L), \quad m_{P}=\operatorname{rk}(P) / n_{P},
$$

thus $m_{P}$ and $n_{P}$ are the multiplicity and the degree of the corresponding irreducible representation of $\mathbb{C} S$. It is known that $m_{P} \geq n_{P}$ for all $P$ [4]. Obviously, for the principal central primitive idempotent $P=(1 /|X|) J$ of the algebra $\mathbb{C} S$ we have $m_{P}=n_{P}=1$.

For an arbitrary field $F$, the semisimplicity of the algebra $F S$ was studied in [5]. It was proved that it is semisimple if and only if the characteristic of the field $F$ does not divide the number

$$
\operatorname{Fr}(X, S)=|X|^{|S|} \frac{\prod_{r \in S} d_{r}}{\prod_{P \in \mathcal{P}} m_{P}^{n_{P}^{2}}}
$$

where $\mathcal{P}$ is the set of all non-principal central primitive idempotents of the algebra $\mathbb{C} S$. This number is called the Frame number of the scheme $(X, S)$.

A scheme $(X, S)$ is called thin, if $d_{r}=1$ for all $r \in S$. In this case there exists a regular group $G \leq \operatorname{Sym}(X)$ such that $S$ coincides with the 2-orbits of $G$, i.e. the orbits of the componentwise action of $G$ on the set $X \times X$. (In this case the sets $X$ and $G$ can be naturally identified and the algebra $F S$ becomes the group algebra $F G$.) Exactly the same construction produces a scheme $(X, S)$ for an arbitrary transitive group $G \leq \operatorname{Sym}(X)$. One can prove that such a scheme is primitive if and only if the group $G$ is primitive.

\section{References}

1. Bannai, E., Ito, T.: Algebraic Combinatorics. I. Benjamin-Cummings, Menlo Park (1984) 
2. Dade, E.C.: Block extensions. Ill. J. Math. 17, 198-272 (1973)

3. Evdokimov, S.A.: Schurity and separability of associative schemes. Doctor of Sciences Thesis, St. Petersburg State University (2005)

4. Evdokimov, S., Ponomarenko, I.: Two inequalities for the parameters of a cellular algebra. Zap. Nauchnykh Seminarov POMI 240, 82-95 (1997). English translation: J. Math. Sci., New York 96(5), 3496-3504 (1999)

5. Hanaki, A.: Semisimplicity of adjacency algebras of association schemes. J. Algebra 225, 124-129 (2000)

6. Hanaki, A., Yoshikawa, M.: On modular standard modules of association schemes. J. Algebraic Combin. 21, 269-279 (2005)

7. Narkiewicz, W.: Elementary and Analytic Theory of Algebraic Numbers. Springer Monographs in Mathematics. Springer, Berlin (2004)

8. Peeters, R.: On the $p$-ranks of the adjacency matrices of distance-regular graphs. J. Algebraic Combin. 15, 127-149 (2002)

9. Weisfeiler, B. (ed.): On Construction and Identification of Graphs. Lecture Notes in Mathematics, vol. 558. Springer, Berlin (1976)

10. Zieschang, P.-H.: Theory of Association Schemes. Springer Monographs in Mathematics. Springer, Berlin (2005) 